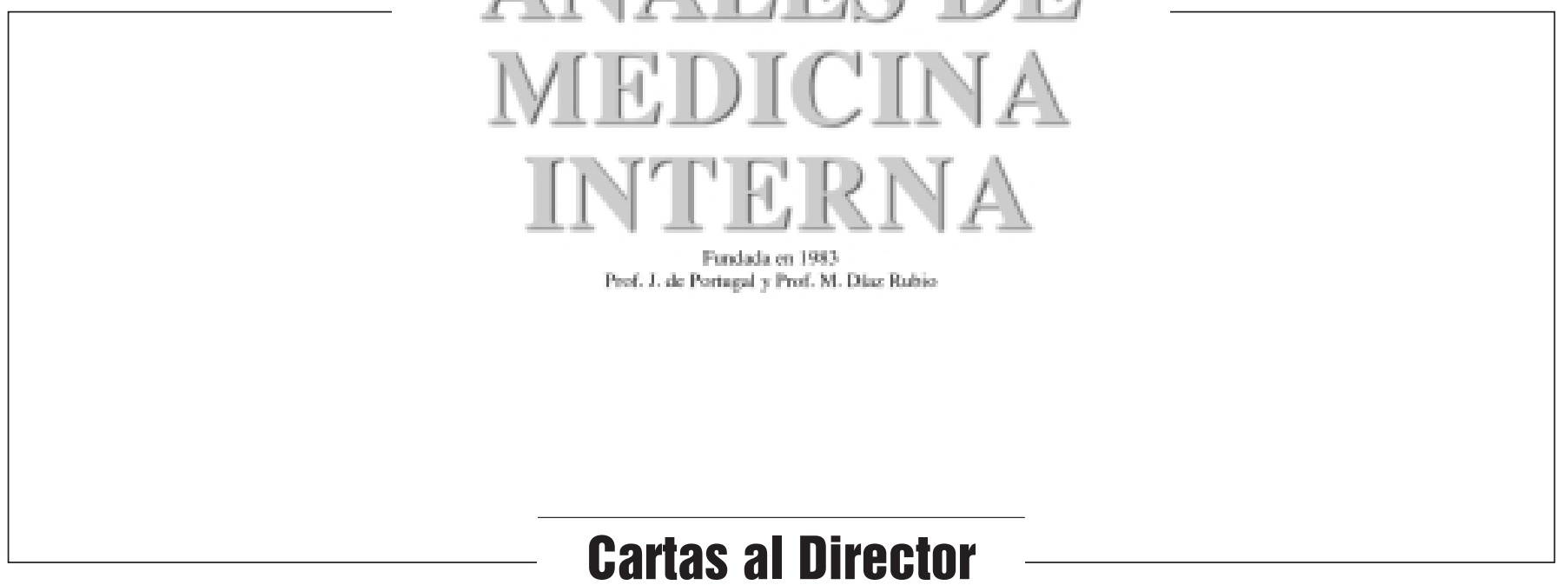

\section{Disección espontánea de la arteria carótida interna}

\section{Sr. Director:}

La disección espontánea de la arteria carótida (DEAC) es una entidad relativamente infrecuente (con una incidencia acumulada estimada de 2,5 casos por cada 100.000 habitantes) (1), si bien puede representar el 10-25\% de las causas de ictus en pacientes jóvenes (2). Presentamos un caso de DEAC.

Una mujer de 32 años presentó bruscamente un cuadro de hemiparesia (de predominio crural) y paresia facial izquierdas y disartria, sin antecedente traumático. Cuando acudió al Servicio de Urgencias de nuestro Hospital, 24 horas después, había remitido parte de la sintomatología y sólo presentaba paresia facial izquierda. En la exploración física al ingreso destacaba borramiento del surco nasogeniano izquierdo y mínima claudicación de extremidad inferior izquierda contra gravedad. La analítica de urgencia, radiografía de tórax, electrocardiograma y la tomografía computarizada (TC) craneal (sin contraste) realizadas a su ingreso fueron normales. El proteinograma, hormonas tiroideas, anticuerpos antinucleares, anti-ADN, serología de VIH, estudio de hipercoagulabilidad (anticuerpos anticardiolipina, anticoagulante lúpico, factor V Leiden, proteínas C y S, antitrombina III) resultaron negativos o dentro de la normalidad así como el estudio de líquido cefalorraquídeo (incluyendo serologías de lúes y Borrelia). El ecocardiograma transtorácico, Holter-ECG y ecografía-doppler de troncos supraaórticos extracraneales no demostraron hallazgos patológicos. En el electroencefalograma realizado destacaba una actividad lenta e irritativa temporal derecha. La resonancia magnética (RM) objetivó múltiples focos de hiperseñal en región de ganglios basales derechos, córtex insular derecho e hipocampo derecho, en probable relación con lesión isquémica. Ante la negatividad del resto de estudios se realizó una arteriografía cerebral que demostró afilamiento y obstrucción de la arteria carótida interna derecha en su porción supraclinoidea, sugestiva de disección arterial, con buen funcionamiento del polígono de Willis y de las anastomosis piales para el hemisferio cerebral derecho. Con estos hallazgos se inició la anticoagulación con heparina sódica y posteriormente con acenocumarina. La evolución posterior de la paciente ha sido favorable sin presentar nuevas recurrencias y persistiendo únicamente mínima paresia facial inferior izquierda. La angio-resonancia magnética (angioRM) realizada al cabo de tres meses de tratamiento demostró
(Fig. 1) disminución persistente y uniforme del calibre de la arteria carótida interna derecha, con calibre normal del bulbo y ausencia de flujo en segmento distal de la misma.

Aunque su causa última permanece desconocida, actualmente se piensa que en los pacientes con DEAC subyace un trastorno del tejido conectivo $(3,4)$ que condiciona un arteriopatía de base sobre la que actuarían diversos factores desencadenantes (tabaco, hipertensión arterial, traumatismos o movimientos de rotación/hiperextensión del cuello) (4). La mayor incidencia de casos de DEAC en pacientes con trastornos del tejido conectivo (síndrome de Ehlers-Danlos tipo IV, síndrome de Marfan, osteo-

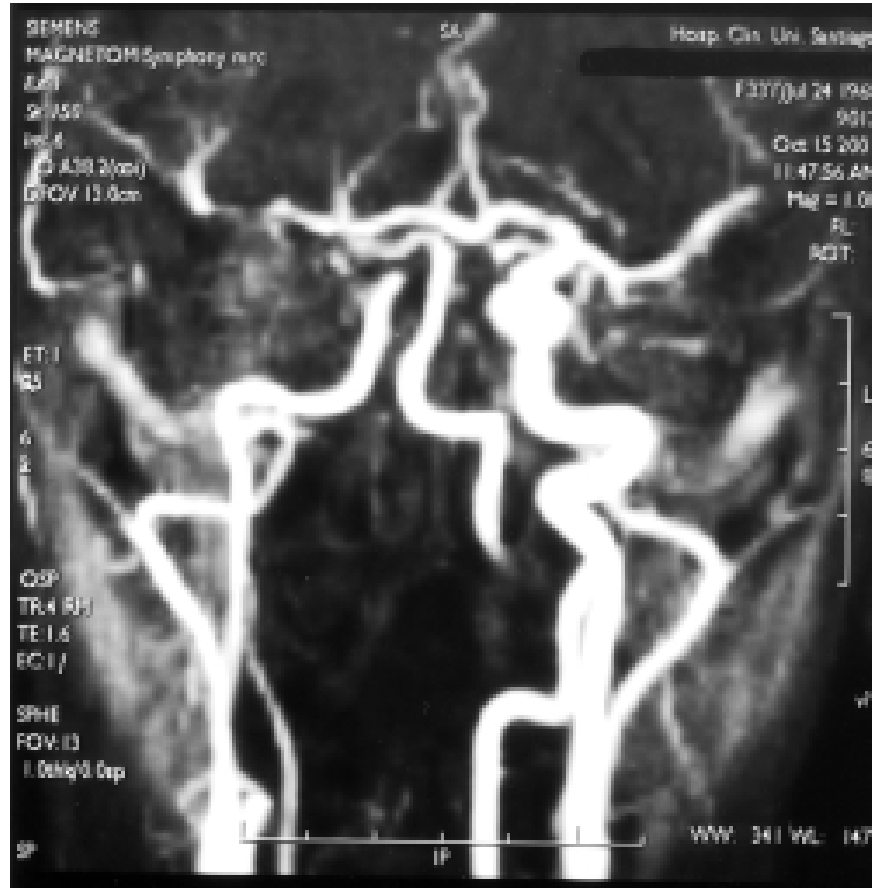

Fig. 1. Angio-resonancia magnética (angio-RM) a los tres meses de tratamiento anticoagulante. Se aprecia disminución persistente y uniforme del calibre de la arteria carótida interna derecha, con calibre normal del bulbo y ausencia de flujo en segmento distal de la misma. 
génesis imperfecta tipo I, degeneración quística de la capa media, displasia fibromuscular,etc) parecen avalar esta teoría (3-5). Asimismo se han descritos casos recurrentes y con agregación familiar $(6,7)$. La forma de presentación típica se basa en la tríada de dolor cervical, síndrome de Horner y manifestaciones de isquemia cerebral dependientes del territorio vascular afectado (4). El diagnóstico se basa en la realización de arteriografía cerebral aunque las nuevas técnicas de angio-RM presentan igual eficacia y facilitan el seguimiento evolutivo además de ser menos invasivas (8). Cabe señalar que hasta en un tercio de los casos, como sucede en el que presentamos, las técnicas ecográficas no son diagnósticas de DEAC(9). El tratamiento de elección se basa en la anticoagulación (inicialmente con heparina y luego con acenocumarina) reservando otros procedimientos (angioplastia percutánea con colocación de stent o cirugía de derivación) para pacientes en los que haya fracasado el tratamiento médico $(4,10)$.

\section{J. Campos Franco, M. R. Alende Sixto, J. A. Castiñeira Mou- renza ${ }^{1}$, A. González Quintela}

Servicios de Medicina Interna y ${ }^{I}$ Radiología. Hospital Clínico. Complejo Hospitalario Universitario. Santiago de Compostela

1. Schievink WI, Mokri B, Whisnant JP. Internal carotid artery dissection in a community: Rochester, Minnesota, 1987-1992. Stroke 1993; 24: 1678-1680.

2. Ducrocq X, Lacour JC, Debouverie M, Bracard S, Girard F, Weber M Accidents vasculaires cérébraux ischémiques du sujet jeune: étude prospective de 296 patients âgés de 16 à 45 ans. Rev Neurol (Paris) 1999; 155: $575-582$.

3. Brandt T, Orberk E, Weber R, Werner I, Busse O, Müller BT, Wigger F, Grau A, Grond-Ginsbach C, Hausser I. Pathogenesis of cervical artery dissections. Association with connective tissue abnormalities. Neurology 2001; 57: 24-30

4. Schievink WI. Spontaneous dissection of the carotid and vertebral arteries. N Engl J Med 2001; 344: 898-906.

5. Schievink WI, Björnsson J, Piepgras DG. Coexistence of fibromuscular dysplasia and cystic medial necrosis in a patient with Marfan's syndrome and bilateral carotid artery dissections. Stroke 1994; 25: 2492-2496.

6. Bassetti C, Carruzzo A, Sturzenegger M, Tuncdogan E. Recurrence of cervical artery dissection: a prospective study of 81 patients. Stroke 1996; 27: 1804-1807

7. Schievink WI, Michels VV, Mokri B, Piepgras DG, Perry HO. A familial syndrome of arterial dissections with lentiginosis. N Engl J Med 1995; 332: 576-579.

8. Djouhri H, Guillon B, Brunereau L, Lévy C, Bousson V, Biousse V, Arrivé L, Tubiana JM. MR Angiography for the long-term follow-up of dissecting aneurysms of the extracranial internal carotid artery. Am J Roentgenol 2000; 174: 1137-1140.

9. Sturzenegger M, Mattle HP, Rivoir A, Baumgartner RW. Ultrasound findings in carotid artery dissection: analysis of 43 patients. Neurology 1995; 45: 691-698.

10. Schievink WI. The treatment of spontaneous carotid and vertebral artery dissections. Curr Opin Cardiol 2000; 15: 316-321.

Paciente con poliartritis séptica y osteomielitis multifocal por diseminación hematógena de Estafilococo aureus

\section{Sr. Director:}

La osteomielitis multifocal es una de las manifestaciones más graves e inusuales de la infección diseminada por Estafilococo aureus, pues la presentación más habitual es monoarticular y en edad pediátrica $(1,2,5,6)$. Creemos de interés comunicar el caso de un varón de 54 años diabético no insulín-dependiente, mal controlado, con afectación micro y macroangiopática. Ingresa por un cuadro de algias generalizadas de predominio en esqueleto axial, malestar y fiebre. Tres meses antes ingresó en UCI por IAM no Q evolucionando sin complicaciones, a excepción de una flebitis supurativa en antebrazo izquierdo, que se resolvió espontáneamente. Al mes de su alta comienza con artromialgias difusas, molestias cérvico-lumbares y malestar general. Fue valorado por diversos facultativos con RMN cervical y dorsolumbar, sin hallazgos. Mes y medio después se añade al cuadro dolor e hinchazón de la cara externa de la rodilla derecha y fiebre elevada $\left(39,5^{\circ} \mathrm{C}\right)$. Es diagnosticado de bursitis séptica, con cultivo positivo para estafilococo dorado en líquido articular y en hemocultivos seriados simultáneos, con igual sensibilidad antibiótica. Se trató ambulatoriamente con amoxiclavulánico durante 15 días con ligera mejoría. Tras la supresión antibiótica y el empeoramiento de los síntomas, le prescriben esteroides 1 $\mathrm{mg} / \mathrm{kg} /$ día, por sospecha de polimialgia reumática durante otras dos semanas. Tres días más tarde acude a nuestro hospital. El paciente se encontraba apirético con marcada postración y dolor intenso a la movilización lumbar y cervical, con signos flogóticos en carpo izquierdo. En la analítica destacaba leucocitosis (14.000) con neutrofilia y VSG 101. En los hemocultivos seriados: se aísla de nuevo estafilococo dorado en cuatro botellas con igual sensibilidad antibiótica que los previos. Se descarta endocarditis ante la ausencia de soplos y ecocardiografía transtorácica y transesofágica sin alteraciones y se inicia tratamiento con cloxacilina i.v. durante 32 días a dosis elevadas, que tras reacción alérgica se sustituye por ciprofloxacino $750 \mathrm{mg} / 12$ hora y rifampicina $600 \mathrm{mg}$ diarios de forma prolongada. La gammagrafía ósea con Tc-99 muestra multiples zonas de hipercaptación a nivel de columna lumbar(L5-S1), columna cervical (C5-C6), carpo izquierdo, sacroilíacas y omóplato derecho. En las nuevas RMN se aprecia: afectación de los cuerpos vertebrales a nivel C5-C6 con discitis intervertebral, espondilitis en L5 con componente de partes blandas intrarraquídeas y paravertrebrales. Irregularidades en escafoides, piramidal, cúbito, trapecio y primer metacarpiano, con cambios inflamatorios y derrame articular (artritis). Pese a la llamativa mejoría general sigue quejándose de dolores intensos en carpo derecho y esqueleto axial. Dos semanas después inicia una cuadro de ciatalgia e impotencia funcional, observándose en TAC y RNM: abceso paralumbar a nivel de L5-S1. Se deriva a neurocirugía, practicandose laminectomía L4-L5 con extirpación del tejido de granulación y discectomía subtotal con franca mejoría subsiguiente.

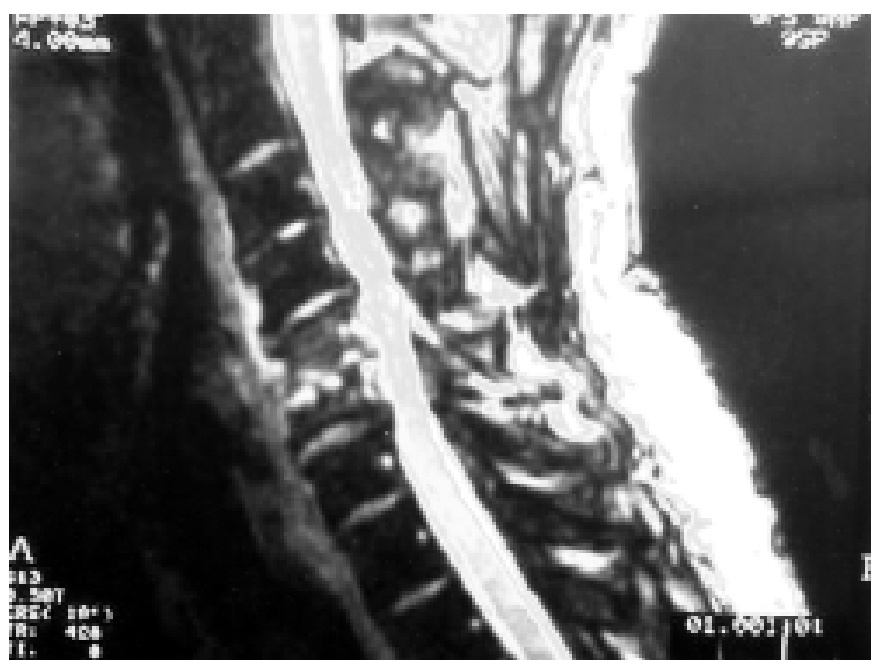

Fig. 1. 
La frecuencia de complicaciones secundarias a bacteriemia por Estafilococo aureus (endocarditis o infección metastásica) es elevada (entre 11 y 53\%) (2,3). Un número cada vez mayor de infecciones bacteriémicas están provocadas por catéteres $(4,9)$. Existe un mayor riesgo de padecer la infección en aquellos pacientes con diabetes, alcoholismo, sida, neoplasias, hemodiálisis, antecedentes de cirugía, ADVP y enfermedades reumáticas (sobre todo artritis reumatoide) preexistentes, a las cuales se atribuyen muchas veces los síntomas de artritis séptica $(3,6)$, o uso de corticoides sistémicos (9). La osteomielitis hematógena que afecta a los cuerpos vertebrales se caracteriza por su inicio insidioso, vaga sintomatología y ocasional compresión medular (4). En nuestro caso el comienzo es tórpido con un periodo de latencia prolongado (entre 1 y 3 meses después de la tromboflebitis). La presentación multifocal es más frecuente en lactantes con afectación de huesos largos predominantemente $(4,5)$, mientras que en adultos son excepcionales los casos recogidos en la literatura $(2,10)$. La artritis séptica por estafilococo se puede dar a cualquier edad, aunque su máxima incidencia es en lactantes y adolescentes, generalmente conduce a severa destrucción articular con alta morbilidad y en el $95 \%$ de los casos la afectación es monoarticular $(1,4,5,7)$. Tan sólo en un $5-10 \%$ de las artritis sépticas la afectación es poliarticular; lo que refleja la bacteriemia y una resistencia disminuida a la infección $(1,6)$.

El diagnóstico debe ser rápido y certero, realizando hemocultivos seriados incluso en pacientes afebriles con sospecha fundada, y el tratamiento antibiótico precoz e intenso para evitar destrucción osteoarticular y complicaciones avanzadas (7). En nuestro caso el tratamiento antibiótico inicial inadecuado y la posterior utilización empírica de esteroides en un paciente diabético, pudieron contribuir a desarrollar complicaciones escasamente referenciadas en la literatura. El sistema inmunológico innato es crucial para defender al huésped contra la infección por estafilococo, mientras que sus componentes más específicos (linfocitos $\mathrm{T}$ y $\mathrm{B}$ y sus productos) son nocivos para el huésped, y actúan de mediadores en el daño articular $(4,7)$.

Queremos enfatizar la necesidad de considerar el diagnostico de metástasis sépticas osteoarticulares en pacientes inmunodeprimidos con antecedentes de flebitis séptica, y cuadros febriles polimiálgicos, sin una clara etiología. A veces el antecedente de flebitis previa pasa desapercibido para el propio paciente, como sucedió en nuestro caso y solo tras recabar información reiterada ante hemocultivos positivos, emerge en la memoria del enfermo.

\section{P. Paricio Núñez, Ma A. Medranda Gómez, A. Tovar Martí- nez, F. Rosique Gómez, V. Buendía Carrillo}

Servicio de Medicina Interna. Hospital Los Arcos. Santiago de la Ribera, Murcia

1. Perry CR, Septic arthritis, Am J Orthop 1999; 28: 168-78.

2. Savvidis E, Parsch K. Hematogenous multifocal osteomyelitis. Orthopade 1997; 26: 879-88.

3. Franklin D. Lowy, MD, Sthaphylococcus aureus infections. N Eng J Med 1998; 20: 520-530.

4. Dagan R. Management of acute hematogenous osteomyelitis and septic arthritis en the pediatric patient. Pediatr Infect Dis J 1993; 12: 88-93.

5. Christiansen P, Fredereksen B, Glazowski J, Scavenius M, Knudsen FU. Epidemilologic, bacteriologic, and long-term follow-up data of children with acute hematogenous osteomyelitis and septic arthritis: a ten year review. Pediatr Orthop B 1999; 8: 302-305.

6. Dubost JJ, Sobrier M, Sauvezie B. Pyogenic arthritis in adults. Joint Bone Spine 2000; 67: 11-21.

7. James W. Anne E. Enfermedades infecciosas. Artritis infecciosa, 1998: 1150-1157.

8. Espinosa Brito A, Martínez Cabrera J. Osteomelitis de la clavícula y artritis séptica esternoclavicular como complicación de la caterización de la vena subclavia. Rev Esp Clin 1990; 5: 38.

9. Menéndez González JA, Martínez Gorantián S, Jazpe Garaicoechea A, Jáuregui Goldis Guardu R. Múltiples complicaciones de la terapeutica con corticoides. Rehabilitación 1990; 24: 129-135.

10. Jaanvaloyas M, Admetla M, Monreal M, Dolzc. S. aureus multiple osteomyelitis after prolonged latency period. An Med Interna (Madrid) 1990; 7 (Supl. 3): 162-3: 163.

\section{Infarto esplénico embólico}

\section{Sr. Director:}

El infarto esplénico puede estar producido por la existencia de diversos trastornos hematológicos, siendo los más frecuentes la metaplasia mieloide, policitemia vera, enfermedades mieloproliferativas, linfomas y leucemias, anemias hemolíticas y otras hemoglobinopatías (1). Sin embargo, la causa más frecuente es la enfermedad tromboembólica, fundamentalmente debido a una fibrilación auricular, generalmente sin tratamiento de anticoagulación y también puede ser producido por la liberación de embolias sépticas en el seno de diversos procesos infecciosos, como la endocarditis. Otras causas posibles son el aneurisma de la aorta abdominal y de la arteria celiaca, trombosis de la arteria o vena esplénica y trombosis portal, aunque también se ha comunicado en otros muchos procesos $(2,3)$.

Presentamos brevemente un nuevo caso estudiado recientemente por nosotros.

Mujer de 73 años de edad, con antecedentes personales de diabetes mellitus en tratamiento con insulina desde hace 16 años, hipertensión arterial desde hace 6 años en tratamiento con dieta sin sal, arritmia cardíaca en tratamiento con digoxina desde hacía cinco años y prótesis de cadera izquierda dos años atrás.

Consultó por presentar desde hacía cinco días un cuadro de dolor abdominal de características cólicas, localizado en el hemiabdomen superior izquierdo. En las últimas horas el dolor se hacía más intenso y se irradiaba al hombro izquierdo. Se acompañaba de náuseas sin vómitos, no fiebre ni alteraciones en las deposiciones.

A la exploración física, la paciente estaba consciente y orientada, bien perfundida e hidratada. Auscultación cardíaca: Tonos arrítmicos en fibrilación auricular, ligero soplo sistólico de carácter eyectivo. Auscultación pulmonar: normal. Abdomen: blando, doloroso sobre todo en el hemiabdomen superior izquierdo, ligeramente distendido sin contractura muscular ni organomegalias, ruidos intestinales normoactivos. Puño percusión renal bilateral negativa.

Laboratorio. Hemograma: leucocitos: $8.000 \mathrm{~mm}^{3}, \mathrm{Ht}^{\mathrm{o}}: 37,2 \%$, hemoglobina de 12,1 gramos/dl, 270.000 plaquetas, actividad de protrombina del $96 \%$.

En la bioquímica destacaba una glucemia de $262 \mathrm{mg} / \mathrm{dl}$, amilasemia de $39 \mathrm{U} / \mathrm{l}$, resto normal. Orina elemental normal.

ECG: Fibrilación auricular con respuesta ventricular controlada, trastornos difusos de la repolarización ventricular.

Radiografía de tórax: ateroma aórtico calcificado, cardiomegalia, no condensaciones pulmonares.

TAC abdominal: bazo de 12 centímetros, se observan dos lesiones hipodensas bien definidas, de bordes lisos y morfología triangular, sin captación periférica, sugerentes como primera posibilidad de infartos esplénicos (Fig. 1).

Ecocardiograma: No patología valvular, no crecimiento de cavidades ni derrame pericárdico.

La enferma recibió tratamiento con heparina de bajo peso 


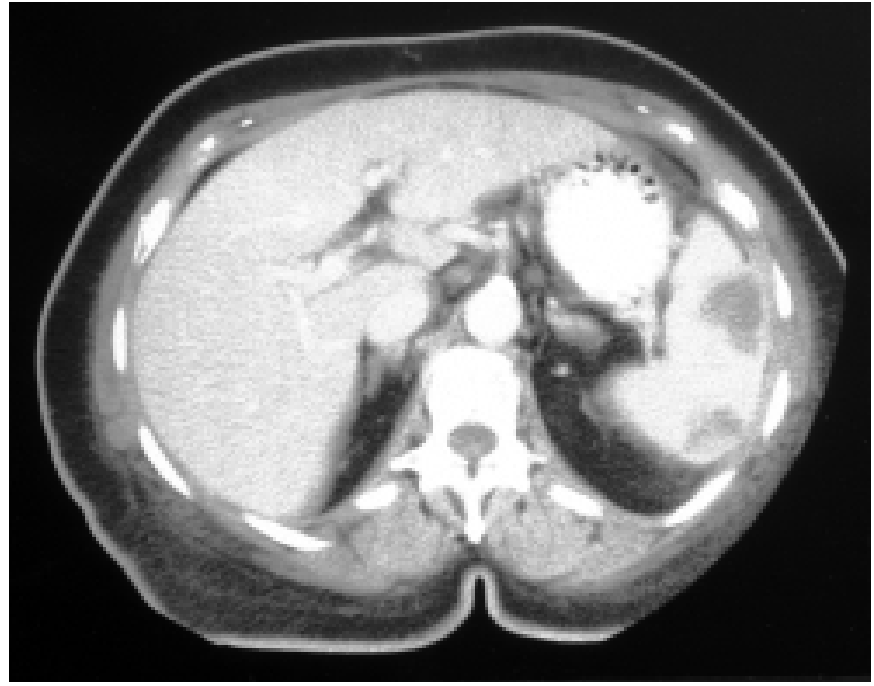

Fig. 1. Se observan 2 lesiones hipodensas en el bazo de morfología triangular, sugerentes de infartos esplénicos.

molecular y posteriormente acenocumarol, evolucionado favorablemente, siendo dada de alta.

En una revisión efectuada tres semanas más tarde la enferma se encontraba bien y no refería dolor abdominal.

El infarto esplénico es una de las causas de dolor agudo o crónico localizado en el hipocondrio izquierdo, aunque también puede presentarse como síndrome febril y en ocasiones como un cuadro constitucional $(4,5)$. En un porcentaje importante de los casos no existen síntomas. La presencia de fiebre suele guardar relación con la existencia de procesos embólicos (4).

Los medios diagnósticos empleados son la ecografía abdominal, que mostrará áreas triangulares hipoecóicas de localización periférica . La tomografía axial computarizada abdominal (TAC) pondrá de manifiesto la existencia de diversas áreas hipodensas periféricas y que no se realzan con el contraste $(4,6)$.

Es fundamental realizar un ecocardiograma, generalmente transesofágico para descartar diversas patologías cardiacas y también la existencia de material embólico en la aorta torácica.

El tratamiento es inicialmente médico, mediante analgésicos y anticoagulación. La cirugía debe reservarse para los casos en que existan complicaciones, tales como el absceso esplénico y la ruptura del bazo o el diagnóstico sea impreciso.

F. Marcos Sánchez, I. Albo Castaño, F. Árbol Linde, A. Viana Alonso, F. M. Gómez Soto, A. Durán Pérez-Navarro

Servicio de Medicina Interna. Hospital Nuestra Señora del Prado. Talavera de la Reina. Toledo

1. Bauer T, Haskins G, Armitage J. Splenic rupture in patients with hematologic malignancies. Cancer 1981; 48: 2729-2733.

2. Agolini SF, Shah KT, Goodreau JJ, McLoughlin TM Jr, Sinclair MC. Splenic infarction caused by a large thoracic aortic thrombus. J Vasc Surg 1997; 26: 1069-1072.

3. Miller FH, Ma JJ. Total splenic infarct due to Aspergillus and AIDS. Clin Imaging 2002; 25: 57-59.

4. Nores M, Phillips EH, Morgenstern L, Hiatt JR. The clinical spectrum of splenic infarction. Am Surg 1998; 64: 182-188.

5. Cuquerella J, Ferrer L, Rivera P, Tuset JA, Medina E, Pamos S, et al. Infarto esplénico. Rev Esp Enf Ap Diges 1996; 88: 447-449.

6. Beeson MS. Splenic infarct presenting as acute abdominal pain in an older patient. J Emerg Med 1996; 14: 319-322.
Síndrome febril intermitente como manifestación inicial de un hemangioma cavernoso gigante hepático

\section{Sr. Director:}

Los hemangiomas cavernosos son los tumores hepáticos benignos más frecuentes. Su prevalencia en la población general varía entre $0,5-7 \%$, afectando predominantemente a mujeres (1). La mayor parte de los hemangiomas hepáticos son asintomáticos, por lo que su diagnóstico suele ser incidental. Sin embargo, aquellos con diámetro superior a $4 \mathrm{~cm}$, hemangiomas cavernosos gigantes, pueden tener expresividad clínica: dolor en hipocondrio derecho, hemoperitoneo por rotura del mismo o asociarse a coagulación intravascular diseminada (síndrome de KasabachMerrit) (2,3).

A continuación exponemos el caso de una paciente con hemangioma cavernoso gigante hepático que se manifestó como síndrome febril intermitente. Se trata de una mujer de 49 años de edad, sin antecedentes de interés, una hermana había sido intervenida previamente de malformación venosa hepática, que consulta por fiebre intermitente de varias semanas de evolución. La paciente había presentado varios episodios febriles que duraban 3-4 días, autolimitados, acompañados de afectación del estado general, sin otros síntomas localizadores del foco febril. La exploración física no mostraba hallazgos relevantes salvo dolorimiento en hipocondrio derecho a la palpación. Presentaba leucocitosis de 21.400 células $/ \mathrm{mm} 3$, sin neutrofilia, proteína $\mathrm{C}$ reactiva de $6,63 \mathrm{mg} / \mathrm{dL}$ sin otras alteraciones en el hemograma y bioquímica. La radiografía de tórax fue normal. El hemocultivo y urocultivo fueron negativos. Asimismo, el rosa de bengala a Brucella, otros antígenos febriles y serología para los distintos virus de la hepatitis fueron negativos. Los marcadores tumorales (alfafetoproteína, antígeno carcinoembrionario) estaban dentro de los valores normales. La ecografía abdominal mostraba lesiones hepáticas múltiples, hiperecogénicas y polilobuladas. Se realizó TAC dinámico en sus fases arterial, portal y portal tardía mostrando 6 lesiones en ambos lóbulos hepáticos, la mayor en el izquierdo, de $12 \mathrm{~cm}$ de diámetro, con un comportamiento dinámico con contraste de realce inicial periférico de las lesiones, extendiéndose progresivamente al centro para rellenarlas por completos (Fig. 1). Estos hallazgos se confirmaron en la RMN hepática que presentaba múltiples lesiones focales hepáticas, de distinto

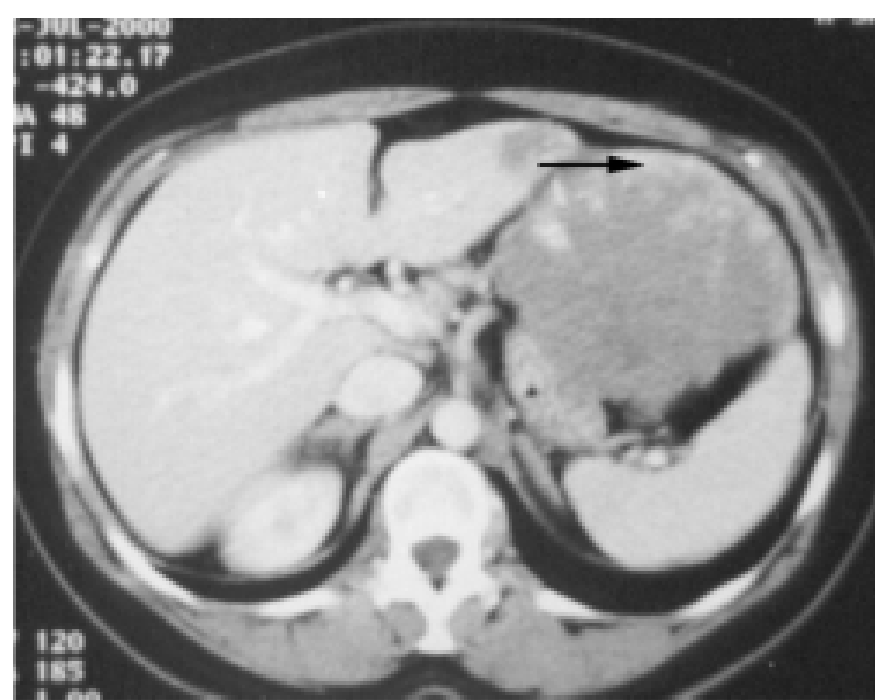

Fig. 1. TAC abdominal dinámico que muestra gran masa en lóbulo hepático izquierdo, que se realza con contraste (flecha). 
tamaño, con contornos lobulados, con hiposeñal respecto al parénquima en las imágenes potenciadas en T1, haciendose hiperintensas en las secuencias potenciadas en $\mathrm{T} 2$.

Una vez que se conoció el resultado del TAC/RNM abdominal y se descartó origen infeccioso del proceso, se instauró tratamiento con antinflamatorios no esteroideos. La paciente permaneció apirética y asintomática. Ulteriormente se realizó segmentectomía (segmento II del lóbulo hepático izquierdo) y tumorectomía a nivel del lóbulo hepático derecho). El análisis histológico de la pieza quirúrgica mostró microscópicamente múltiples nódulos de contornos mal definidos, constituidos por espacios vasculares no anastomosantes, algunos muy dilatados, tapizados por células endoteliales aplanadas y apoyados sobre estroma fibroso, apreciándose trombos intravasculares en diferentes estadios de organización, propio de hemangioma cavernoso. La evolución posterior fue favorable, desapareciendo el síndrome febril intermitente, permaneciendo la paciente asintomática.

Los hemangiomas hepáticos son tumores benignos que se suelen diagnosticar de forma incidental, dado que suelen ser asintomáticos. Sin embargo, los hemangiomas cavernosos gigantes (aquellos con un tamaño superior a los $4 \mathrm{~cm}$ ) suelen manifestarse generalmente con dolorimiento en hipocondrio derecho $\mathrm{y}$, rara vez, ocasionan complicaciones como hemoperitoneo o coagulación intravascular diseminada. Existen algunos casos descritos en la literatura que se manifiestan por fiebre elevada, siendo debido a trombosis dentro del hemangioma y ocurriendo, generalmente, en aquellos de mayor tamaño (4-7).

Ecográficamente, muestran un patrón hiperecogénico, aunque los de mayor tamaño pueden ser heterogéneos por necrosis irregular de algunas zonas siendo, entonces difíciles de diferenciar de procesos malignos. No obstante, en el TAC con contraste suelen presentar un patrón típico con captación periférica inicial y distribución posterior al centro de la lesión (8).

Los hemangiomas de pequeño tamaño y aquellos que cursan asintomáticos no requieren tratamiento quirúrgico, bastando para su manejo un seguimiento periódico, dado que usualmente no incrementan su tamaño durante muchos años. Sin embargo, los de gran tamaño y los que presentan manifestaciones clínicas, precisan ser resecados, que se asocia con escasa morbimortalidad (9). La embolización selectiva de la arteria hepática previa disminuye las complicaciones de la cirugía, dado que decrece el riesgo de hemorragia intraoperatoria (10).

\section{A. García Ordóñez, M. Cabello Ramírez ${ }^{1}$ I. Hierro Mar- tín ${ }^{2}$}

Servicio de Medicina Interna. ${ }^{1}$ Unidad de Digestivo. ${ }^{2}$ Servicio de Anatomía Patológica. Hospital de Antequera. Málaga

1. Ishak KG, Rabin L. Benign tumors of the liver. Med Clin North Am 1975; 59: 995-1013.

2. Golfieri R, Minguzzi MT, Lalli A, Soro A, Totaro C, Giampalma E, Gavelli G. The spontaneous rupture of a hepatic cavernous hemangioma. A case report and review of the literature. Radiol Med 1994; 88 : 315-9.

3. Shimizu M, Miura J, Itoh H, Saitoh Y. Hepatic giant cavernous hemangioma with microangiopathic hemolytic anemia and consumption coagulopathy. Am J Gastroenterol 1990; 85: 1411-3.

4. Pol B, Disdier P, Le Treut YP, Campan P, Hardwigsen J, Weiller PJ. Inflammatory process complicating giant hemangioma of the liver: report of three cases. Liver Transpl Surg 1998; 4: 204-7.

5. Fenster LF, Freeny PC, Beebe HG. Cavernous hemangioma of the liver presenting with fever. Successful treatment with prednisone. West J Med 1978; 129: 138-40.

6. Utrilla Ayala D, Robles Frías A, Ortiz del Río CA, León Jímenez EM, Lozano de León F. Fiebre prolongada y tumoración hepática. Rev Clin Esp 1999; 199: 539-40.

7. Taillandier J, Alemanni M, Veyssier P, Smadja C, Manigand G, Franco
D. Giant hemangioma of the liver disclosed by fever and an inflammatory syndrome. Ann Med Interne (París) 1995; 146: 319-21.

8. Choi BI, Han MC, Park JH, et al. Giant cavernous hemangioma of the liver: CT and MR imaging in 10 cases. AJR Am J Roentgenol 1989; 152: 1221-6.

9. Hanazaki K, Kajikawa S, Matsushita A, et al. Hepatic Resection of Giant Cavernous Hemangioma of the Liver. J Clin Gastroenterol 1999; 29: 257-60.

10. Suzuki H, Nimura Y, Kamiya J, et al. Preoperative transcatheter arterial embolization for giant cavernous hemangioma of the liver with consumption coagulopathy. Am J Gastroenterol 1997; 92: 688-71.

\section{Resolución espontánea de un quiste tímico primario}

\section{Sr. Director:}

Los tumores mediastínicos constituyen un grupo heterogéneo de enfermedades neoplásicas, congénitas e inflamatorias. En el mediastino anterior se diagnostican hasta el $50 \%$ de estos tumores y la mayoría son timomas o formaciones quísticas consideradas inicialmente como benignas (1). A pesar de su benignidad, en todos los casos se recomienda una resección quirúrgica para confirmar el diagnóstico y evitar las complicaciones relacionadas con su ruptura, fistulización, infección, hemorragia, malignización o compresión de las estructuras vecinas por su crecimiento, considerando como excepcional una evolución como la de nuestro paciente $(2,3)$.

Varón de 45 años, exfumador de 20 paquetes/año sin antecedentes patológicos de interés. En los últimos 10 días refería unas molestias retroesternales inespecíficas y febrícula sin tos, disnea, escalofríos o síndrome constitucional. En la exploración física presentaba un buen estado general sin acropaquias, adenopatías periféricas palpables o signos de insuficiencia cardiaca. La auscultación cardiaca y respiratoria fueron normales. La radiografía de tórax demostró la presencia de una masa homogénea y bien delimitada de aproximadamente $6 \mathrm{~cm}$ de diámetro mayor en el mediastino antero-superior que confirmó la TC y la resonancia nuclear magnética (RNM), con un contenido líquido que sugería la presencia de un quiste multiloculado a nivel del timo, sin signos de invasión o adenopatías mediastínicas y con un pequeño derrame pleural derecho (Fig. 1). El hemograma, la coagulación y la bioquímica fueron normales, con una valor de la VSG de 36 $\mathrm{mm} / \mathrm{h}$ y valores normales del antígeno carcinoembrionario (CEA), alfa-fetoproteína, Beta-HCG, Beta-2 microglobulina, inmunoglobulinas y hormonas tiroideas o negativos de las serologías para el VIH y la hidatidosiss. El electrocardiograma, la ecografía abdominal y la broncoscopia fueron normales junto a un test de la tuberculina negativo.

Al mes del diagnóstico de un posible quiste tímico, se realizó una esternotomía sin observar lesiones quísticas en el mediastino y donde el timo era macroscópicamente normal, con una zona de menor consistencia y coloración amarillenta de $1 \mathrm{~cm}$, de aspecto residual en cuya histología solo se evidenció una mínima inflamación crónica localizada sin signos de malignidad. A los 8 meses de la intervención el paciente estaba asintomático y con una radiografía de tórax normal.

Los quistes tímicos son infrecuentes y constituyen menos del $3 \%$ de todos los tumores del mediastino anterior. Existen quistes congénitos y adquiridos, estos últimos después de un proceso inflamatorio local o en asociación a neoplasias como el linfoma, el seminoma o el carcinoma tímico $(1,3)$. Más del $50 \%$ permanecen asintomáticos en el tiempo y son diagnosticados de forma 


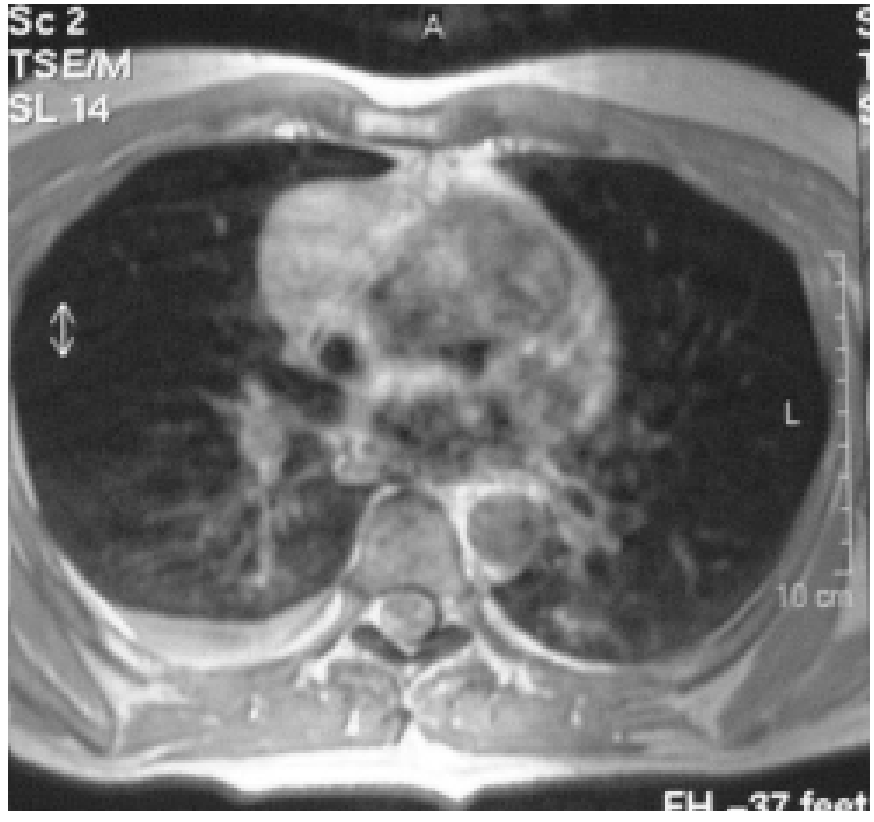

Fig. 1. Resonancia nuclear magnética. Quiste tímico antero-superior sin signos de malignidad y con pequeño derrame pleural derecho.

casual en la radiografía, al aparecer en el mediastino anterior una tumoración homogénea, redondeada, bien delimitada, uni o multiloculada, con una pared fina y un contenido líquido o semisólido que se visualiza mejor con la TC o la RNM $(1,4)$. La posibilidad de aparición de complicaciones, su asociación a determinadas neoplasias o la imposibilidad de diferenciarlos de otras neoplasias quísticas del timo obligan a su resección diagnóstica y terapéutica, con la posibilidad de una posterior recidiva en las formas adquiridas (1).

La resolución espontánea de las lesiones quísticas en el mediastino es muy infrecuente y excepcional en el caso del timo (6). Existen muy pocos casos descritos de una regresión de un quiste tímico y esta evolución suele atribuirse a su ruptura por un aumento de las presiones intratorácicas, la digestión enzimática de su pared por la reacción inflamatoria o su erosión por el contacto con estructuras vecinas de mayor consistencia como la carina o el esófago (5). En nuestro paciente, la presencia inicial de síntomas y un pequeño derrame pleural derecho sugiere su ruptura y posterior vaciado con un comportamiento poco habitual que debería ser considerado en pacientes seleccionados y sin otras alteraciones acompañantes, al permitir una actitud más conservadora a la espera de su evolución a corto plazo cuando existe un mayor riesgo quirúrgico.

\section{Haro Estarriol, X. Baldó Padró, M. Rubio Goday, F. Sebastián Quetglas}

Sección de Neumología. Servicios de Medicina Interna y Cirugía Torácica. Hospital Universitario de Girona "Doctor Josep Trueta”. Girona

1. Strollo DC, Rosado CLC, Jett JR. Primary mediastinal tumors. Part 1: Tumors of the anterior mediastinum. Chest 1997; 112: 511-22.

2. Morgenthaler TI, Brown LR, Colby TV, Harper CM, Coles DT. Thymoma. Mayo Clin Proc 1993; 68: 1110-23.
3. Komaki R, Putnam JB, Moon D, Cox JD. Thymic neoplasms. Curr Opin Oncology 1997; 9: 158-60.

4. Choi YW, McAdams HP, Jeon SC, Hong EK, Kim YH, Im JG et al. Idiophatic multilocular thymic cyst: CT features with clinical and histopathologic correlation. AJR 2001; 177: 881-5.

5. Martin KW, Siegel MJ, Chesna E. Spontaneous resolution of mediastinal cysts. AJR 1988; 150: 1131-2.

6. Leibman AJ, Morehouse HT, Ziprkowski M. Spontaneous rupture of a thymic cyst demonstrated by computed tomography. J Comput Assist Tomogr 1984; 8: 550-2.

\section{Amiloidosis sistémica primaria y miocardiopatía dilatada}

\section{Sr. Director:}

La amiloidosis sistémica idiopática o primaria (AL) es debida al depósito extracelular de una proteina amiloide

compuesta por fracciones de inmunoglobulina de cadenas ligeras y que es producida por una población monoclonal de células plasmáticas (1). La afectación por sustancia amiloide afecta a casi todos los órganos de la economia y su depósito en el corazón es un hecho común y causa de morbilidad frecuente por arritmias, alteraciones del sistema de conducción aurículo-ventricular y coronariopatías (2-4). Mientras que el $30 \%$ de los pacientes con amiloidosis AL presentan en su evolución una cardiopatía clínicamente evidente debida a una miocardiopatía restrictiva infiltrativa $(2,5,6)$, el hallazgo de una miocardiopatía dilatada se ha descrito ocasionalmente $(7,8)$. Por ello creemos de interés presentar un paciente con amiloidosis $\mathrm{AL}$ en el que predominaban las manifestaciones de insuficiencia cardiaca congestiva debida a una miocardiopatía dilatada.

Varón de 75 años, fumador de 20-30 cigarrillos al día y con antecedentes de EPOC de 20 años de evolución que cursa con una obstrucción moderada al flujo aéreo (FEV1 1,60 litros, 58\% del teorico). Ingresa en nuestro centro por iniciar 2 meses antes edemas progresivos en extremidades inferiores, disnea de esfuerzo creciente y ortopnea. En la exploración física destacaba una PA 110/50, $\mathrm{T}^{\mathrm{a}}$ axilar $36^{\circ}$, palidez cutáneo-mucosa, edemas con fóvea en extremidades inferiores, IY y RHY positivos, hepatomegalia dura de $12 \mathrm{~cm}$ bajo reborde costal, tonos cardiacos rítmicos a 90 lx'con soplo sistólico 3/6 plurifocal y estertores crepitantes teleinspiratorios en $2 / 3$ inferiores de ambos hemitórax. La radiología torácica evidenció una cardiomegalia importante con signos de hipertensión veno-capilar pulmonar y pinzamiento de ambos senos costodiafragmáticos. En el ECG se observó una onda $\mathrm{T}$ negativa en cara ánterolateral. En la analítica destacaba una VSG de $75 \mathrm{~mm} 1^{\mathrm{a}}$ hora, Hb $10 \mathrm{~g} / \mathrm{dl}$, Hto 32\%, VCM $87 \mathrm{fl}$, HCM 27 pg, creatinina 4,9 umol/L, GOT 56 U/L, GPT 74 U/L, GGT 465 U/L, FA 435 U/L, LDH 210 U/L, proteinuria de 9,2 $\mathrm{g} / 24$ horas y un proteinograma con una hipoalbuminemia (48\%) y presencia de un componente homogéneo de migración gamma que correspondía a una paraproteina de cadenas ligeras Lambda. La ecografía abdominal confirmó una hepatomegalia homogénea sin LOES, con esplenomegalia discreta , sin otras alteraciones. El ecocardiograma-doppler evidenció una miocardiopatía dilatada $(\mathrm{DTDVI}=64 \mathrm{~mm}, \mathrm{DTSVI}=54 \mathrm{~mm}, \mathrm{~F}$. acortamiento $=16 \%)$ con una insuficiencia mitral secundaria de grado severo, una hipertensión pulmonar moderada $(39 \mathrm{mmHg})$ y una fracción de eyección del 34\% (Fig. 1). El estudio EMG mostró una polineuropatía sensitivo-motora moderada de carácter axonal y de predominio en extremidades inferiores. Una fibrogastroscopia puso de manifiesto una lesión polipoide prepilórica cuya biopsia fue diagnósti- 


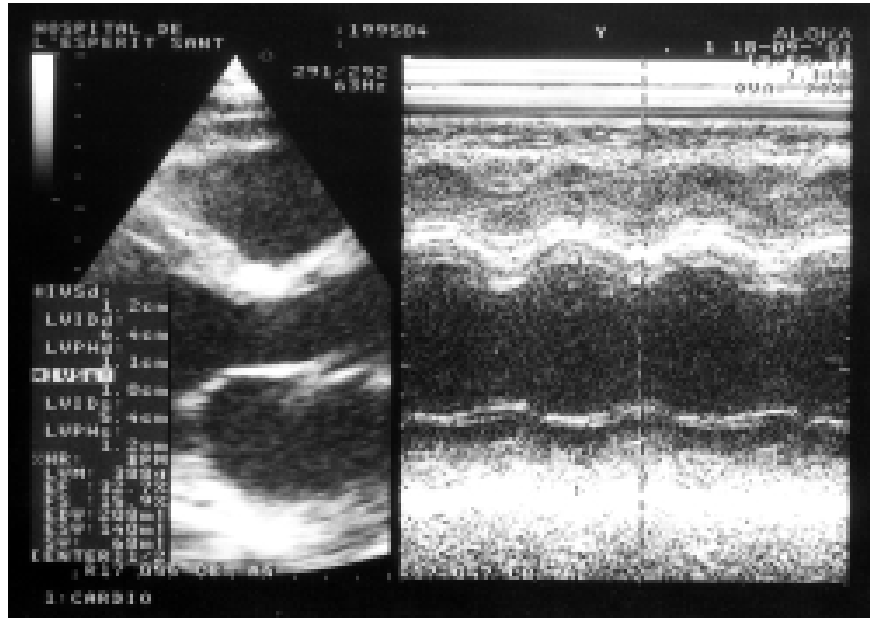

Fig. 1. Ecocardiograma-doppler: Cavidades ventriculares izquierdas dilatadas con una función ventricular deprimida.

ca de amiloidosis, lesion que tambien se halló en las biopsias efectuadas en mucosa rectal y que se identificó como amiloidosis tipo AL. La seriada ósea radiológica descartó lesiones líticas y el estudio citológico medular mostró una plasmocitosis inferior al $10 \%$. El paciente inició tratamiento con melfalán y prednisona, pero debido al progresivo deterioro de la función renal fue remitido a centro de referencia para iniciar programa de hemodialisis.

La afectación cardiaca en la amiloidosis es frecuente y su presencia es el factor pronóstico más importante y lo que determina la supervivencia en los pacientes con dicha enfermedad $(1,2)$. En el miocardio, la sustancia amiloide se deposita en el intersticio, entre los miocitos y la membrana basal vascular, en forma de agregados nodulares con engrosamiento de las paredes ventriculares, del septo interventricular y del tejido valvular. Ello produce una insuficiencia cardiaca congestiva rápidamente progresiva, en la que la ecografía-doppler muestra un patrón restrictivo con cavidades ventriculares de tamaño normal o reducido, disfunción diastólica, disminución del gasto cardiaco $(1,5)$, y una imagen de textura miocárdica anormal en forma de granulado brillante (1). Tambien se pueden observar bloqueos aurículo-ventriculares por infiltración del sistema de conducción y la aparición de arritmias supra y ventriculares por depósito de amiloide en aurículas. La afectación por amiloidosis coronaria obstructiva intramural provoca angor, y tambien es frecuente observar hipotensión ortostática por infiltración del sistema nervioso autónomo, fenómenos embólicos periféricos y patología valvular cardiaca $(3,4)$. En los casos en que se ha efectuado $(4,9,10)$, la biopsia endomiocárdica muestra el depósito extracelular de proteína fibrilar amiloide en el intersticio miocárdico, en forma de infiltrados reticulares, desorganización de la arquitectura tisular normal y atrofia de los miocitos. En general, la amiloidosis es causa de miocardiopatia infiltrativa restrictiva junto con la sarcoidosis y las enfermedades de Gaucher y de Hurler $(5,6)$. El aumento del grosor muscular ventricular y septal cardiacos dificulta la función ventricular, con una disminución del volúmen diastólico y del gasto cardiaco (6). La miocardiopatía dilatada en la amiloidosis se ha descrito esporádicamente $(7,8)$, y en este caso la ecografía-doppler muestra una dilatación de las cavidades ventriculares con un patrón de disfunción contráctil sistólica y afectación valvular con insuficiencias mitral y/o tricuspídea de intensidad severa. El pronóstico de los pacientes con amiloidosis y afectación cardiaca es sombrio (8), con un promedio de vida inferior a los 6 meses, aunque tras los tratamientos con melfalán, doxorubicina y prednisona o incluso el trasplante de médula ósea, la supervivencia media puede alcanzar los 18 meses, y un 5\% de los pacientes pueden sobrepasar los 10 años $(3,8,11)$. En algunos casos seleccionados, y en la fase final de la amiloidosis, el trasplante cardiaco puede mejorar la calidad de vida y la supervivencia (12).

\section{E. Rodríguez, T. Poblet, R. López ${ }^{1}$, T. Ribera}

Servicios de Medicina Interna $y^{\prime}$ Laboratorio. Hospital de L'Esperit Sant. Santa Coloma de Gramenet, Barcelona

1. Kyle RA. Amyloidosis. Circulation 1995; 91: 1269-1271.

2. Wynne J, Braunwald E. Miocardiopatías y miocarditis. En: Braunwald E ed. Tratado de Cardiología. Mc Graw-Hill Interamericana. México. 1997: p. 1535-1598

3. Grogan M, Gertz MA, Kyle RA, Tajik AJ. Five or more years of survival in patients with primary systemic amyloidosis and biopsy-proven cardiac involvement. Am J Cardiol 2000; 85: 664-665.

4. Mueller PS, Edwrds WD, Gertz MA. Symptomatic ischemic heart disease resulting from obstructive intramural coronary amyloidosis. Am J Med 2000; 109: 181-188.

5. Spyrou N, Foale R. Restrictive cardiomyopathies. Curr Opin Cardiol 1994; 9: 344-348.

6. Kushwaha SS, Fallon JT, Fuster V. Restrictive cardiomyopathy. N Eng J Med 1997; 336: 267-276.

7. Bristow MR, Bohlmeyer TJ, Gilbert EM. Dilated cardiomyopathy. En: Alexander RW ed. The Hearth. Mc Graw-Hill. New York. 1998: 20392055.

8. Laraki R. L’amylose cardiaque. Revue générale. Rev Med Interne 1994; 15: 257-267.

9. Scully RE, Mark EJ, Mc Neely WF, Ebeling SH, Ellender SM. Case records of the Massachusetts General Hospital. Weekly clinicopathological exercises. Case 3-2000: A 66-year-old woman with diabetes, coronary disease, orthostatic hypotension and the nephrotic syndrome. $\mathrm{N}$ Eng J Med 2000; 342: 264-273.

10. Kingman AMY, Pereira NL. Cardiac amyloidosis. J S C Med Assoc 2001, 97: p. 201-206.

11. Kyle RA, Gertz MA, Greipp PR et al. Long-term survival ( 10 years or more) in 30 patients with primary amyloidosis. Blood 1999; 93: 10621066.

12. Gertz MA. Amyloidosis: Recognition, prognosis and conventional therapy. En: Educational Program of American Society of Hematology Congress. Miami. 1999; 339-357.

\section{FE DE ERRATAS}

En el volumen 20, número 6 de la revista Anales de Medicina Interna, en la página 307, correspondiente al artículo: "Enfermedad de Mondor: estudio de dos formas topográficas", aparece un error en el título en inglés donde pone "Monsor's disease: study of two topographic localizations", siendo el título correcto "Mondor's disease: study of two topographic localizations" 